www.nature.com/hr

\title{
A long-term, comprehensive exercise program that incorporates a variety of physical activities improved the blood pressure, lipid and glucose metabolism, arterial stiffness, and balance of middle-aged and elderly Japanese
}

\author{
Terukazu Kawasaki ${ }^{1,6}$, Corbet V Sullivan ${ }^{2}$, Naomi Ozoe ${ }^{3}$, Hidehiko Higaki ${ }^{4}$ and Junya Kawasaki ${ }^{5}$
}

A 6-month, twice-a-week exercise program emphasizing swimming was conducted for 11 men (57-73 years) and 24 women (51-68 years). The control group comprised 11 male (59-70 years) and 11 female (53-70) volunteers. The exercise program significantly improved the systolic and diastolic blood pressure (SBP/DBP) and lipid and glucose metabolism, with no change in the controls. Brachial-ankle pulse wave velocity (baPWV), as an index of systemic arterial stiffness, was measured during medical examinations before and after each exercise session using a volume-plethysmographic apparatus. SBP and DBP of the extremities were significantly decreased after exercise, but did not change in the controls. Average baPWV decreased significantly in the exercise group, from $1661 \pm 50$ to $1581 \pm 40 \mathrm{~cm}$ per sec. No change was seen in the controls. The sway path of the center of balance was analyzed using a force plate. The length of postural sway, the length of postural sway per sec and the area of postural sway were measured with eyes open and eyes closed, and the rectangular area was calculated. The eyes open/eyes closed ratio (Romberg sign) was also calculated. All parameters of body sway were significantly lower after 6 months in the exercise group, with no change in the controls. The Romberg sign did not change for either group. In addition to promoting better health, as shown by the clinical data, this type of exercise program improves balance function, which could help prevent falls of the elderly.

Hypertension Research (2011) 34, 1059-1066; doi:10.1038/hr.2011.81; published online 14 July 2011

Keywords: arterial stiffness; balance function; blood pressure; lipid metabolism; water exercise

\section{INTRODUCTION}

With increased longevity, the quality of life and general health of the older segments of society have become important topics of discussion and research. Physical exercise programs are commonly promoted for the prevention and treatment of lifestyle-related health conditions. ${ }^{1-3}$ Good physical condition allows older persons to live independently and continue to participate as valuable members of society, while greatly reducing the health-care burden.

Blood pressure (BP), lipid and glucose metabolism, and arterial stiffness are all well-documented causes of morbidity and mortality, which are related to lifestyle and the quality of life (QOL).

Hypertension is well known as a major risk factor for the development of arteriosclerosis. Sustained hypertension is one of the highest risk factors for cardiovascular accidents (CVA). The mortality and morbidity rates by CVA are proportionally increased with elevation of $\mathrm{BP}^{4,5}$ The fact that physical activity is effective for the prevention and treatment of mild or moderate hypertension, carbohydrate intolerance and dyslipidemia has been clearly demonstrated, and exercise is recommended in the Guidelines for the Management of Hypertension JSH $2009 .{ }^{6}$ Aerobic exercise at $\sim 50 \%$ of maximum oxygen uptake $\left(\mathrm{VO}_{2} \mathrm{max}\right)$ has been recommended as the most effective non-pharmacological treatment for individuals with high BP. ${ }^{4}$

Lipid and glucose metabolism, as well as arteriosclerosis, worsen with advancing age. Arterial stiffening is a manifestation of arteriosclerosis, leading to thickening and loss of elasticity in the arterial wall. One of the indicators of arterial stiffness is pulse wave velocity (PWV),

\footnotetext{
${ }^{1}$ Center for Health and Sports Science, Department of Medicine and Clinical Science, Graduate School of Medical Sciences, Kyushu Sangyo University, Fukuoka, Japan; ${ }^{2}$ Language Education and Research Center, Kyushu Sangyo University, Fukuoka, Japan; ${ }^{3}$ Kyushu Sangyo University Swimming Club, Kyushu Sangyo University, Fukuoka, Japan; ${ }^{4}$ Faculty of Engineering, Kyushu Sangyo University, Fukuoka, Japan and ${ }^{5}$ Kawasaki Cardiovascular Clinic, Department of Medicine and Clinical Science, Graduate School of Medical Sciences, Kyushu Sangyo University, Fukuoka, Japan

${ }^{6}$ Current address: 3-2-1 Kashiihama \#111, Higashi-ku 813-0016, Japan.

Correspondence: Dr T Kawasaki, Center for Health and Sports Science, Department of Medicine and Clinical Science, Graduate School of Medical Sciences, Kyushu University, 3-2-1 Kashiihama \#111, Higashi-ku 813-0016, Japan.

E-mail: tkawas63@crocus.ocn.ne.jp
}

Received 9 February 2011; revised 31 March 2011; accepted 30 April 2011; published online 14 July 2011 
most often estimated by measuring carotid-femoral PWV. Owing to its time efficiency and technical simplicity, the measurement of brachial-ankle PWV (baPWV) has been recommended for use in the clinical setting. Its validity, reproducibility and high correlation with aortic PWV obtained by a catheter tip manometer have been previously reported. ${ }^{7}$ Values of baPWV have been found to correlate with carotid-femoral PWV values ${ }^{8}$ and have also been shown to be significantly associated with the presence and extent of atherosclerotic vascular diseases 9,10 and the cardiovascular risk factors of healthy adolescents. ${ }^{11}$ Moreover, baPWV has been found to be a marker of the efficacy of therapies intended to achieve regression of arterial stiffness. ${ }^{12}$ Recently, the measurement of PWV has received much attention as a marker of arterial stiffness, elasticity and extension, which can be easily demonstrated by form PWV/ankle-brachial pressure index (ABI) measurement. ${ }^{13}$

Most studies have been focused on walking, jogging, cycling or ergometer (stationary bicycle or rowing machine), which are commonly recommended aerobic exercises. Swimming and walking in water have been promoted as beneficial for the treatment of cardiovascular diseases and metabolic abnormalities, notably for the prevention and treatment of hypertension and CVA. Water has the added benefit that it protects against injuries from falls. To date, however, very few researchers have conducted clinical studies on their effectiveness. ${ }^{14,15}$ Hirofumi Tanaka (Sports Medicine) has done extensive research on the cardiovascular effects of exercise, and concluded that 'the CHD risk profile is more favorable in swimmers than in sedentary counterparts and that swim training results in the lowering of some $\mathrm{CHD}$ risk factors'. However, he cautions that the benefit of swimming may be lower than that for land-based exercises. ${ }^{14}$

Postural balance is often impaired in the elderly, and can result in falls of the elderly that result in reduced activity of daily life and QOL, with many elderly persons becoming bedridden. ${ }^{16-19}$ Such falls are one of the main causes for elderly persons becoming permanently bedridden. Lord et al. ${ }^{16}$ carried out a 1-year exercise trial that examined balance, strength and falls of older women. However, this study was conducted on a floor where several types of injuries, such as bone fractures and ankle twists, could have occurred by unexpected falls. Exercise on hard floors is problematic for elderly persons in general, especially those with pre-existing back or joint pain or muscle weakness. Water exercise, however, eliminates these problems; thus it is safer and allows most persons to participate in exercise programs, in spite of their physical limitations. Hara et al. ${ }^{19}$ investigated the effects of exercise in water on the balance of middle-aged women in a study that was limited to only 8 weeks and did not include other parameters, such as relating exercise to lifestyle modification or arteriosclerosis.

To confirm the effects of exercise on BP, glucose and lipid metabolism, arterial stiffness and balance of middle-aged and elderly, clinically healthy Japanese persons, this 6-month, parallel case-control study was conducted using a comprehensive exercise program that incorporates a variety of physical activities.

\section{METHODS}

\section{Participants}

A convenience sample of clinically healthy men and women between the age of 50 and 70 years volunteered for placement in a water-exercise group or control group (no exercise change to their current routine). Recruitment was carried out by means of a local newspaper advertisement or by leaflets placed on notice boards in community centers near our university.

All participants, both from the exercise and control groups, met the following inclusion criteria: (1) no history of symptomatic stroke, cardiac, renal or other serious disease, (2) no current physical exercise routine; the participants in the exercise group met two additional criteria: (3) sufficient time to participate in the exercise sessions on a regular basis for the full 6 months, and (4) strong motivation to participate in the study.

Approval for the study was obtained from the Research Ethics Committee of Kyushu Sangyo University and written informed consent was obtained from each participant.

\section{Study protocol}

The study was conducted from mid May to mid November to reduce the effects of seasonal variation ${ }^{20-22}$ for groups of up to 15 participants each year over a period of 3 years. All participants received a thorough medical examination that included medical history, routine physical examination, electrocardiogram (ECG) and blood test. A medical examination was conducted 2 weeks before the beginning of the first exercise session to gather baseline data, and a second examination was conducted at the end of the study to gather post-training data for comparison.

The participants in the exercise group committed to attending the exercise sessions twice each week for 6 months. None were able to swim 25 meters at the beginning of the exercise program, and most of them had to learn how to swim. The members of both groups were asked not to make major lifestyle changes during the period of the study. We confirmed that the control group participants did not have a current physical exercise routine.

Each exercise session took about 2 hours and comprised (1) measurement of $\mathrm{BP}$, heart rate (HR) and body weight (BW); (2) stretching for 5-10 min, then riding a stationary bicycle for $20 \mathrm{~min}$; (3) land-based muscle strengthening exercises suitable for older persons for $10 \mathrm{~min}$; (4) walking in the swimming pool for $40 \mathrm{~min}$ with/against a water stream generator $\left(0.9 \mathrm{~m} \mathrm{~s}^{-1}\right)$ that provides additional resistance; (5) swimming for $20 \mathrm{~min}$ in water maintained at $30.5^{\circ} \mathrm{C}$; and (6) measurement of BP, HR and BW. Two instructors supervised each exercise session. The exercise intensity was adjusted for each individual to $50 \%$ of the maximal oxygen uptake as calculated by the Karvonen formula: Target $\mathrm{HR}=[(220-$ Age $)-$ Resting HR $] \times 0.5+$ Resting HR. As the exercise capacity of the participant improved, the exercise routine was modified to maintain the appropriate intensity.

\section{Measurements}

At the time of placement into the exercise or control group, each participant filled out a questionnaire on their suitability for enrollment in the study and additional questions about lifestyle, including eating, smoking and drinking habits, as well as family and medical histories.

The following items were measured before and after the 6-month program for both the exercise and control groups.

\section{Anthropometric measurements}

Height, BW and body fat (Tanita Comp., Tokyo, Japan) were measured for both groups at medical examinations carried out before and at the end of the study. BW was also measured for the exercise group before and after each exercise session.

\section{$B P$ and $H R$}

Both parameters were measured on three different occasions: (1) in a sitting position for $10 \mathrm{~min}$ during the medical examination. Three consecutive BP readings were obtained by mercury sphygmomanometer and averaged for the analysis, (2) in a supine position at the time of measurement of baPWV on the bilateral arm. The average value of both arms was used for the analysis. Supine HR was taken from the ECG recording; (3) both parameters were also measured before and after every exercise session using an automated sphygmomanometer (Colin, Komaki, Japan). To estimate the effects of the 6-month physical exercise program on these parameters, we compared the BP and HR values obtained at the pre- and post-exercise medical examinations. Furthermore, the average $\mathrm{BP}$ and $\mathrm{HR}$ values obtained for the first three and the last three exercise sessions were compared to determine the effects of the long-term exercise regimen. 


\section{Blood test}

Venous blood samples were obtained after overnight fasting in a relaxed sitting position to determine plasma glucose, hemoglobin $\mathrm{A}_{1 \mathrm{C}}$, serum lipid profile and electrolytes. Analyses were carried out by an autoanalyzer at the Clinical Research Center Co. Fukuoka, Japan (CRC).

\section{Urinary excretion of electrolytes}

On the second morning, voiding urine (SMU) samples were obtained at each medical examination. Urinary concentrations of sodium $(\mathrm{Na})$, potassium $(\mathrm{K})$ and creatinine $(\mathrm{Cr})$ were measured, and 24-h excretions of $\mathrm{Na}$ and $\mathrm{K}$ were estimated by our established method. ${ }^{23-27}$ To confirm the consistency of the participants' diet over the study period, urinary concentrations of calcium (UCa) and magnesium (UMg) were also measured, and the $\mathrm{UCa} / \mathrm{UMg}$ ratio was calculated. Urinary urea nitrogen (UN) was measured and the UN/UCr the ratio was calculated before and after the study period for each participant to roughly estimate the stability of the nutrient intake. Urine parameters were also measured at CRC. UCa/UMg ratio and UN/UCr data are not shown.

\section{baPWV and ABI}

The baPWV was used as an index of systemic arterial stiffness. We measured baPWV and ABI at each medical examination using a volume-plethysmographic apparatus (form PWV/ABI, Colin Co., Komaki, Japan). ${ }^{7}$ Measurement was taken in a supine position. Electrodes for PWV were placed on each wrist and each ankle, and a microphone for detecting heart sounds was placed on the left edge of the sternum. The cuffs were connected to a plethysmographic sensor that determines volume pulse form and an oscillometric pressure sensor that measures BP. The pulse waveforms were recorded using a semiconductor pressure sensor (the sample acquisition frequency for PWV was set at $1200 \mathrm{~Hz})^{28,29}$

Trunk control test (TCT) of body sway

The sway path of the center of balance was analyzed using a force plate (FP) developed by one of the authors ( $\mathrm{HH})$ and his laboratory staff. The subject

Table 1 Baseline profile at the first medical examination

\begin{tabular}{lcc}
\hline & Exercise group & Control group \\
\hline No. of subjects & 35 & 22 \\
Men/women & $11 / 24$ & $11 / 11$ \\
Age (years) & $61.5 \pm 0.8$ & $62.6 \pm 0.9$ \\
Height (cm) & $158.9 \pm 1.4$ & $158.6 \pm 1.8$ \\
Body weight (kg) & $60.1 \pm 1.8$ & $57.1 \pm 2.1$ \\
Body mass index $\left(\mathrm{kg} \mathrm{m}^{-2}\right)$ & $23.7 \pm 0.6$ & $22.7 \pm 0.7$ \\
Number of smoking habit (\%) & $6 / 35(17.1)$ & $4 / 22(18.2)$ \\
Number of drinking (\%) & $11 / 35(31.4)$ & $7 / 22(31.8)$
\end{tabular}

Mean \pm s.e.m.

Smoking habit: 5 per day or more.

Drinking habit: $20 \mathrm{~g}$ per day of ethanol or more. stood on the center of the force plate in Romberg posture and measurements were taken over $60 \mathrm{~s}$, once with eyes open and once with eyes closed. The length of postural sway (LPS); LPS per second; the area of postural sway; and the rectangular area, which was the product of the area of the postural sway of the maximal anterior-posterior distance and right-left distance, were calculated. We also calculated the eyes open/eyes closed ratio (Romberg ratio) for these parameters.

\section{Statistical analysis}

All analyses were performed using the Stat View 4.5 software package (HULINKS, Tokyo, Japan). Values are expressed as mean \pm s.e.m. Differences between the water-exercise and control groups were analyzed by unpaired $t$-test. The effects of the 6-month exercise program on the hemodynamic and biochemical parameters were tested by paired $t$-test. Association of the changes in baPWV with those of the other variables was analyzed by calculating Pearson's correlation coefficients. A probability value $<0.05$ was considered to be statistically significant.

\section{RESULTS}

Subject profiles

After the medical examination, 39 volunteers satisfied the inclusion criteria. The data of four members of the exercise group who had $<80 \%$ attendance were censored, leaving the data of 11 men (57-73 years) and 24 women (51-68 years) available for analysis. The control group consisted of 22 volunteers, 11 men (59-69 years) and 11 women (53-70 years).

Profiles of the exercise and control groups at the medical examination before starting the protocol are shown in Table 1 . No significant between-group differences were found in age (range 51-73 years), height, BW, body mass index (BMI), body fat, systolic blood pressure (SBP), diastolic blood pressure (DBP), HR, or the percentage of smokers and drinkers.

In the first year, the exercise group included 14 persons, the second included 13 and the third 8 . In the control group, the participants were 0,20 and 2 in the respective years. Participants taking antihypertensive drugs were four and three in the exercise and control groups, respectively, and one participant in each group was taking drugs for diabetes mellitus or liver disease. A letter was sent to each participant's family doctor asking that the dosage and kind of medicine not be changed during the study period.

The average rate of participation in the twice-weekly exercise sessions was $92.4 \%$, with no difference between men and women.

Changes in BW, BMI, body fat, BP, HR from pre- to post-exercise medical examinations

The results of the baseline and 6-month medical examinations are shown in Table 2. BW, BMI and body fat were significantly decreased

Table 2 Changes in body composition, blood pressure and heart rate at the medical examination and 6 months after water exercise

\begin{tabular}{|c|c|c|c|c|}
\hline & \multicolumn{2}{|c|}{ Exercise group $(\mathrm{n}=35)$} & \multicolumn{2}{|c|}{ Control group $(\mathrm{n}=22)$} \\
\hline & Before & After & Before & After \\
\hline Body weight (kg) & $60.1 \pm 1.8$ & $59.1 \pm 1.7^{* * *}$ & $57.1 \pm 2.1$ & $57.2 \pm 2.0$ \\
\hline Body mass index $\left(\mathrm{kg} \mathrm{m}^{-2}\right)$ & $23.7 \pm 0.6$ & $23.4 \pm 0.5^{* * *}$ & $22.7 \pm 0.7$ & $22.7 \pm 0.6$ \\
\hline$\%$-Fat (\%) & $29.9 \pm 1.1$ & $29.8 \pm 1.0$ & $28.1 \pm 1.1$ & $28.3 \pm 1.1$ \\
\hline Body fat (kg) & $18.2 \pm 1.0$ & $17.8 \pm 0.9 *$ & $16.1 \pm 0.9$ & $16.2 \pm 0.8$ \\
\hline Systolic blood pressure $(\mathrm{mm} \mathrm{Hg})$ & $136.6 \pm 3.2$ & $127.0 \pm 2.7^{* * *}$ & $133.9 \pm 2.3$ & $132.7 \pm 2.5$ \\
\hline Diastolic blood pressure (mm Hg) & $81.0 \pm 1.6$ & $77.5 \pm 1.3^{* * *}$ & $77.8 \pm 1.5$ & $80.3 \pm 1.5$ \\
\hline Heart rate (b.p.m.) & $65.0 \pm 1.1$ & $63.2 \pm 0.8$ & $63.5 \pm 1.1$ & $63.6 \pm 1.4$ \\
\hline
\end{tabular}

Before: medical examination at entry, After: 6 months after water exercise.

${ }^{*} P<0.05,{ }^{* * *} P<0.001$ (vs. before). 
after 6 months in the exercise group, whereas no differences were detected in the control group.

$\mathrm{BP}$ was significantly decreased in the exercise group at all three check points $(P<0.001)$, as shown in Tables 2 and 3: (I) SBP/DBP:

Table 3 Changes in body weight, blood pressure, heart rate (HR) and exercise load in the exercise group

\begin{tabular}{lrc}
\hline & Baseline & After 6 months \\
\hline Values at the start of the exercise session & & \\
Body weight (kg) & $59.9 \pm 1.7$ & $59.3 \pm 1.6^{*}$ \\
Systolic blood pressure (mm Hg) & $134.1 \pm 3.0$ & $123.1 \pm 2.2^{* * *}$ \\
Diastolic blood pressure (mm Hg) & $82.6 \pm 1.8$ & $77.3 \pm 1.7^{* * *}$ \\
HR before stationary bicycle training (b.p.m.) & $78.4 \pm 1.8$ & $77.2 \pm 1.8$ \\
& & \\
& & \\
Values at the end of the exercise session & $59.5 \pm 1.7$ & $58.8 \pm 1.6^{* *}$ \\
Body weight (kg) & $137.6 \pm 3.9$ & $125.0 \pm 2.3^{* * *}$ \\
Systolic blood pressure (mm Hg) & $86.7 \pm 2.4$ & $78.9 \pm 2.0^{* * *}$ \\
Diastolic blood pressure (mm Hg) & $112.7 \pm 2.3$ & $113.3 \pm 1.7$ \\
HR after stationary bicycle training (b.p.m.) & $81.1 \pm 2.3$ & $80.0 \pm 2.3$ \\
HR (b.p.m.) & $52.7 \pm 1.6$ & $64.6 \pm 1.9^{* * *}$ \\
Exercise load (watt) & 0 & $24.1 \pm 3.3^{* * *}$ \\
Rate of increase of exercise load (\%) & $47.7 \pm 3.2$ & $48.5 \pm 2.9$ \\
\%-Increase of HR before and after & & \\
stationary bicycle training (\%) & \\
\hline
\end{tabular}

Baseline: the first day of exercise (about one to two weeks after the first medical examination). ${ }^{*} P<0.05,{ }^{* *} P<0.01,{ }^{* * *} P<0.001$ (vs, before). from $136.6 \pm 3.2 / 81.0 \pm 1.6 \mathrm{~mm} \mathrm{Hg}$ to $127.0 \pm 2.7 / 77.5 \pm 1.3$, (II) from $134.1 \pm 3.0 / 82.6 \pm 1.8 \mathrm{~mm} \mathrm{Hg}$ to $128.9 \pm 2.6 / 77.3 \pm 1.7$ and (III) from $134.1 \pm 3.0 / 82.6 \pm 1.8 \mathrm{~mm} \mathrm{Hg}$ to $123.1 \pm 2.2 / 77.3 \pm 1.7$, respectively. HR was not different at testing points (I) and (III), but decreased significantly at testing point (II).

The average exercise load on the stationary bicycle, as calculated by the Karvonen formula, at the beginning of the program was $47.7 \pm$ 3.2 watts ( $45-75$ watts). It gradually increased to $64.6 \pm 1.9$ watts (55-105 watts) at the end of the program. The rate of increase was $24.1 \pm 3.3 \%$ (Table 3 ).

\section{Blood and urine test}

As shown in Table 4, serum K, total cholesterol (TC), LDL-cholesterol, atherosclerotic index (AI: TC-HDL-C/HDL-C ratio) and fasting blood sugar (FBS) were all significantly decreased in the exercise group, and hemoglobin Alc had a nonsignificant decrease. The 24-h urinary excretions of $\mathrm{Na}$ and $\mathrm{K}$ estimated by SMU, and the $\mathrm{UCa} / \mathrm{UMg}$ and UN/UCr ratios in urine before and after 6 months were not significantly different in the exercise and control groups (data not shown).

\section{baPWV and $A B I$}

SBP and DBP of all four extremities significantly decreased after exercise in the exercise group, but did not change in the control group.

The results of baPWV and ABI, measured by ABI-form, are shown in Table 5. Average baPWV decreased significantly in the exercise group, from $1661 \pm 50$ to $1581 \pm 40 \mathrm{~cm} \mathrm{~s}^{-1}$, whereas no change was

Table 4 Comparison of pre- and post-exercise blood and urine chemistries

\begin{tabular}{|c|c|c|c|c|}
\hline \multirow[b]{2}{*}{ Parameters } & \multicolumn{2}{|c|}{ Exercise group } & \multicolumn{2}{|c|}{ Control group } \\
\hline & Before & After & Before & After \\
\hline \multicolumn{5}{|l|}{ Blood } \\
\hline Red blood cell (per mm³) & $462 \pm 25$ & $441 \pm 24$ & $461 \pm 23$ & $466 \pm 23$ \\
\hline Hemoglobin $\left(\mathrm{g} \mathrm{dl}^{-1}\right)$ & $14.5 \pm 0.2$ & $13.9 \pm 0.2$ & $13.8 \pm 0.2$ & $13.9 \pm 0.2$ \\
\hline Hematocrit (\%) & $43.5 \pm 0.6$ & $42.1 \pm 0.6$ & $42.0 \pm 0.7$ & $42.8 \pm 0.7^{*}$ \\
\hline White blood cell (per $\mathrm{mm}^{3}$ ) & $4960 \pm 267$ & $4990 \pm 221$ & $5013 \pm 321$ & $5300 \pm 327$ \\
\hline Total protein $\left(\mathrm{g} \mathrm{dl}^{-1}\right)$ & $7.1 \pm 0.1$ & $7.1 \pm 0.1$ & $7.0 \pm 0.1$ & $7.1 \pm 0.1$ \\
\hline Serum urea nitrogen $\left(\mathrm{mg} \mathrm{dl}^{-1}\right)$ & $14.5 \pm 0.5$ & $14.4 \pm 0.6$ & $14.8 \pm 0.8$ & $14.8 \pm 0.6$ \\
\hline Serum creatinine $\left(\mathrm{mg} \mathrm{dl}^{-1}\right.$ ) & $0.67 \pm 0.02$ & $0.68 \pm 0.02$ & $0.66 \pm 0.03$ & $0.66 \pm 0.03$ \\
\hline Serum uric acid (mg dl ${ }^{-1}$ ) & $5.3 \pm 0.23$ & $5.2 \pm 0.2$ & $5.1 \pm 0.3$ & $5.4 \pm 0.3 * *$ \\
\hline Serum $\mathrm{Na}\left(\mathrm{mEq} \mathrm{I}^{-1}\right)$ & $141.5 \pm 0.3$ & $141.2 \pm 0.3$ & $142.1 \pm 0.3$ & $142.1 \pm 0.3$ \\
\hline Serum $\mathrm{K}\left(\mathrm{mEq} \mathrm{I}^{-1}\right)$ & $4.34 \pm 0.07$ & $4.57 \pm 0.08^{*}$ & $4.42 \pm 0.08$ & $4.57 \pm 0.12$ \\
\hline Serum $\mathrm{Cl}\left(\mathrm{mEq} \mathrm{I}^{-1}\right)$ & $102.3 \pm 0.4$ & $102.4 \pm 0.3$ & $102.5 \pm 0.5$ & $103.4 \pm 0.4$ \\
\hline Serum Ca $\left(\mathrm{mg} \mathrm{dl}^{-1}\right)$ & $9.2 \pm 0.1$ & $9.2 \pm 0.1$ & $9.2 \pm 0.1$ & $9.3 \pm 0.1$ \\
\hline Serum Mg (mg dl-1) & $2.53 \pm 0.03$ & $2.49 \pm 0.03^{* *}$ & $2.55 \pm 0.05$ & $2.51 \pm 0.03$ \\
\hline Total cholesterol (mg dl-1) & $224.7 \pm 6.4$ & $212.4 \pm 5.2^{* * *}$ & $221.5 \pm 10.0$ & $225.1 \pm 10.5$ \\
\hline HDL-cholesterol (mg dl-1) & $65.3 \pm 2.6$ & $66.6 \pm 2.9$ & $65.9 \pm 4.2$ & $65.8 \pm 3.9$ \\
\hline Triglycerides $\left(\mathrm{mg} \mathrm{dl}^{-1}\right)$ & $104.9 \pm 7.9$ & $99.2 \pm 6.2$ & $106.2 \pm 13.4$ & $94.2 \pm 9.7$ \\
\hline LDL-cholesterol (mg dl $\left.{ }^{-1}\right)$ & $131.8 \pm 5.6$ & $124.3 \pm 5.3^{*}$ & $123.5 \pm 6.3$ & $134.1 \pm 8.3^{* *}$ \\
\hline Atherosclerotic index (TC-HDL-C/HDL-C) & $2.64 \pm 0.17$ & $2.44 \pm 0.19 *$ & $2.63 \pm 0.29$ & $2.72 \pm 0.28$ \\
\hline Atherosclerotic index (LDL-C/HDL-C) & $2.17 \pm 0.13$ & $2.06 \pm 0.16^{\Delta}$ & $2.09 \pm 0.20$ & $2.27 \pm 0.23^{*}$ \\
\hline Creatine phosphokinase $\left(\mid \mathrm{UI}^{-1}\right)$ & $109.8 \pm 9.0$ & $112.4 \pm 10.0$ & $123.3 \pm 16.4$ & $131.5 \pm 21.5$ \\
\hline Fasting blood sugar (mg dl${ }^{-1}$ ) & $104.1 \pm 5.6$ & $96.0 \pm 2.7^{* *}$ & $88.3 \pm 2.1$ & $92.7 \pm 2.0^{* *}$ \\
\hline Hemoglobin $A_{1 c}(\%)$ & $5.7 \pm 0.3$ & $5.4 \pm 0.1^{\Delta}$ & $5.0 \pm 0.2$ & $5.1 \pm 0.2^{*}$ \\
\hline \multicolumn{5}{|l|}{ Urine } \\
\hline Estimated urinary $\mathrm{Na}$ excretion (mEq per day) & $232 \pm 9$ & $228 \pm 9$ & $203 \pm 9$ & $189 \pm 10$ \\
\hline Estimated urinary $\mathrm{K}$ excretion (mEq per day) & $56.8 \pm 3.2$ & $57.9 \pm 2.5$ & $55.8 \pm 2.5$ & $54.0 \pm 3.3$ \\
\hline
\end{tabular}

Abbreviations: C, cholesterol; HDL, high density lipoprotein; LDL, low density lipoprotein; TC, total cholesterol.

${ }^{\Delta} P<0.1,{ }^{*} P<0.05,{ }^{* *} P<0.01,{ }^{* * *} P<0.001$ (vs. before). 
Table 5 Comparison of blood pressure, baPWV and ABI before and after exercise

\begin{tabular}{|c|c|c|c|c|}
\hline & \multicolumn{2}{|c|}{ Exercise group } & \multicolumn{2}{|c|}{ Control group } \\
\hline & Before & After & Before & After \\
\hline Right arm (mm Hg) & $136.8 \pm 3.5$ & $129.0 \pm 2.6^{* *}$ & $136.9 \pm 3.1$ & $135.4 \pm 2.9$ \\
\hline Light arm (mm Hg) & $136.0 \pm 3.5$ & $128.9 \pm 2.6^{* *}$ & $135.8 \pm 3.2$ & $133.5 \pm 2.8$ \\
\hline Light arm (mm Hg) & $81.2 \pm 2.0$ & $76.7 \pm 1.6^{* * *}$ & $78.6 \pm 1.8$ & $79.8 \pm 1.9$ \\
\hline Right baPWV $\left(\mathrm{cm} \mathrm{s}^{-1}\right)$ & $1653.4 \pm 48.9$ & $1573.7 \pm 38.2^{* *}$ & $1650.3 \pm 65.9$ & $1655.9 \pm 62.5$ \\
\hline Left baPWV $\left(\mathrm{cm} \mathrm{s}^{-1}\right)$ & $1668.5 \pm 52.3$ & $1588.0 \pm 41.4^{*}$ & $1696.5 \pm 69.7$ & $1705.3 \pm 68.7$ \\
\hline Mean baPWV $\left(\mathrm{cm} \mathrm{s}^{-1}\right)$ & $1661.0 \pm 50.2$ & $1580.9 \pm 39.6^{* *}$ & $1673.4 \pm 67.6$ & $1680.6 \pm 65.4$ \\
\hline Mean ABI & $1.12 \pm 0.01$ & $1.14 \pm 0.01^{* *}$ & $1.14 \pm 0.01$ & $1.15 \pm 0.01$ \\
\hline
\end{tabular}

Abbreviations: $\mathrm{ABI}$, systolic blood pressure of leg/systolic blood pressure of arm ratio; baPWV, brachial-ankle pulse wave velocity.

${ }^{*} P<0.05,{ }^{* *} P<0.01,{ }^{* * *} P<0.001$ (vs. before)

Table 6 Single correlation coefficients between baPWV and other related variables

\begin{tabular}{|c|c|c|c|}
\hline & baPWV & & $\%$ baPWV \\
\hline $\operatorname{SBP}^{a}(57)^{c}$ & $0.761 * * *$ & $\%-S_{B P a}(57)^{c}$ & $0.555^{* * *}$ \\
\hline $\operatorname{DBP}^{a}(57)^{c}$ & $0.373^{* *}$ & $\%-\operatorname{DBP}^{a}(57)^{\mathrm{c}}$ & $0.370 * *$ \\
\hline $\operatorname{SBP}^{b}(57)^{c}$ & $0.796 * * *$ & $\%-\operatorname{SBP}^{b}(57)^{\mathrm{c}}$ & $0.697 * * *$ \\
\hline $\operatorname{DBP}^{b}(57)^{\mathrm{c}}$ & $0.733^{* * *}$ & $\%-D_{B P}^{b}(57)^{c}$ & $0.600 * * *$ \\
\hline Total cholesterol $(57)^{\mathrm{c}}$ & 0.092 & $\%$-Total cholesterol $(57)^{\mathrm{c}}$ & $0.354 * *$ \\
\hline HDL-cholesterol $(57)^{\mathrm{C}}$ & -0.043 & $\%-H D L-c h o l e s t e r o l(57)^{c}$ & 0.006 \\
\hline LDL-cholesterol $(57)^{\mathrm{c}}$ & 0.028 & $\%$-LDL-cholesterol $(57)^{\mathrm{C}}$ & $0.292^{*}$ \\
\hline Al $(57)^{\mathrm{C}}$ & 0.137 & $\%-A I(57)^{C}$ & 0.218 \\
\hline Fasting blood sugar $(57)^{c}$ & $0.349^{* *}$ & $\%$-Fasting blood sugar $(57)^{\mathrm{C}}$ & $0.477^{* * *}$ \\
\hline Sway with eyes closed & & $\%$-Sway with eyes closed & \\
\hline Total sway ${ }^{d}(46)^{c}$ & 0.233 & Total sway ${ }^{d}(46)^{c}$ & 0.028 \\
\hline Total swaye $(46)^{c}$ & $0.375^{* *}$ & Total swaye $(46)^{c}$ & 0.125 \\
\hline Sway with eyes open & & \%-Sway with eyes open & \\
\hline Total swayd $(46)^{c}$ & 0.047 & Total swayd $(46)^{c}$ & 0.054 \\
\hline Total sway ${ }^{\mathrm{e}}(46)^{\mathrm{c}}$ & 0.111 & Total sway ${ }^{\mathrm{e}}(46)^{\mathrm{c}}$ & 0.131 \\
\hline
\end{tabular}

Abbreviations: AI, (total cholesterol-HDL-cholesterol)/HDL-cholesterol ratio; baPWV, mean brachial-ankle pulse wave velocity; DBP, diastolic blood pressure; SBP, systolic blood pressure. ${ }^{a}$ At the medical examination; ${ }^{\text {bAt the PWV measurement; ' }}$ number of subjects examined; ${ }^{\mathrm{d} T h e}$ product of sway area; ${ }^{\mathrm{e} T h e}$ retangle area. \%-: \%-Change (after 6 months/at the entry) $\times 100$

product of sway area; ${ }^{*}$ The retangle area. $\%-:$ : $\%$-Change (atter 6 mo
$* P 0.05, * * P<0.01, * * * P<0.001$ (baPWV vs, related variables).

seen in the control group, $1673 \pm 68$ and $1681 \pm 66 \mathrm{~cm} \mathrm{~s}^{-1}$. Average $\mathrm{ABI}$ increased significantly in the exercise group, from $1.12 \pm 0.01$ to $1.14 \pm 0.01$, whereas no change was seen in the control group, $1.14 \pm 0.01$ and $1.15 \pm 0.01$.

Correlation coefficients between baPWV and other related variables Single correlation coefficients (CC) between baPWV and other related variables are shown in Table 6. CC between baPWV and BP, especially SBP, were the highest of the parameters measured. No CC between baPWV and any lipid parameter was detected, whereas the CC between baPWV and FBS showed a fairly good relation. On the other hand, little CC was found between baPWV and movements of central gravity, either with eyes closed or open.

\section{TCT of body sway}

Comparison of the balance data of the first and second medical examinations is shown in Figure 1. All parameters of TCT were significantly lower after 6 months in the exercise group, but no significant change was detected in the control group. The Romberg test value did not change for either group.

\section{DISCUSSION}

Lifelong exercise has long been promoted for health maintenance and improving the QOL of the older segments of society. The results of this study showed that a long-term, comprehensive exercise program that incorporated a variety of physical activities improved the BP, lipid and glucose metabolism, arterial stiffness and balance of a group of middle-aged and elderly Japanese.

The main new information provided is as follows: (A) arterial stiffness measured using form PWV/ABI as an index of systemic arterial stiffness, and (B) the use of a FP to test balance before and 6 months after an exercise program and comparison of both exercise and control groups. These parameters have not been examined in previous investigations.

The baPWV of our participants improved significantly over the 6 months of exercise (Table 5), but did not change in the control group. baPWV is important because it reflects arterial stiffness. ${ }^{30,31}$ The participants in our study averaged about 62 years and the BP of the four extremities was high-normal BP or hypertension for most persons in both groups. baPWV was higher than that of an NT group of the same age in another study. ${ }^{9}$ However, BP decreased significantly in our exercise group, but no change was observed in the control group. baPWV has been shown to be affected by glucose and lipid metabolism; ${ }^{10}$ thus, the improvement of baPWV may be related to the BP-lowering effect of the exercise itself and also to the improvements of glucose and lipid metabolism.

We were encouraged that the participants maintained their motivation and strictly adhered to the program, despite the long 6-month protocol. Avoiding boredom from a program becoming routine is important, and thus we added stretching and riding a stationary bicycle to swimming and walking in the pool, which were the main components of the program. It has been reported that maintaining continuous, good human relationships itself decreases BP and promotes lifestyle modification. ${ }^{2}$ The study members formed a unique bond with each other and the instructors over the 6 months of the study, which could have been partially responsible for the improvements in BP. It is noteworthy that most of the participants have continued the exercise program. 

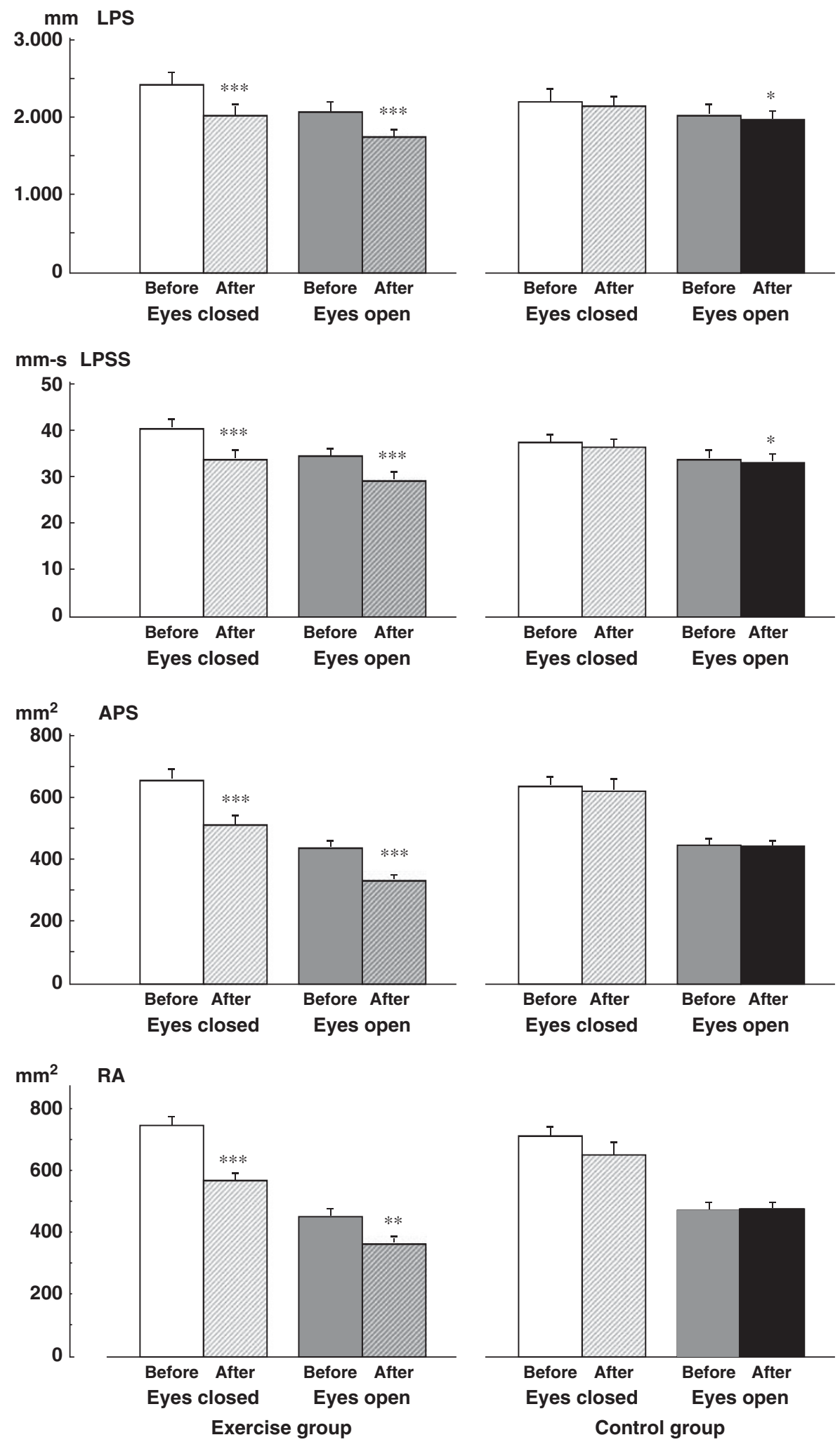

Figure 1 Results of before and after measurement of length of postural sway (LPS), length of postural way per second (LPSS), area of postural sway (APS) and the rectangular area (RA) in the exercise and the control groups. RA was calculated from the length and the width of postural sway. Before: before exercise; After: after 6 months of exercise or 6 months after the first test for the control group. ${ }^{*} P<0.05,{ }^{* *} P<0.01,{ }^{* * *} P<0.001$ (vs. before).

It is generally accepted that physical activity is effective for the prevention and treatment of mild or moderate hypertension, carbohydrate intolerance and dyslipidemia, and aerobic exercise at $\sim 50 \%$ of maximum oxygen uptake $\left(\mathrm{VO}_{2} \mathrm{max}\right)$ has been recommended as the most effective non-pharmacological treatment. ${ }^{1}$ However, very few clinical investigations have been done concerning the beneficial 

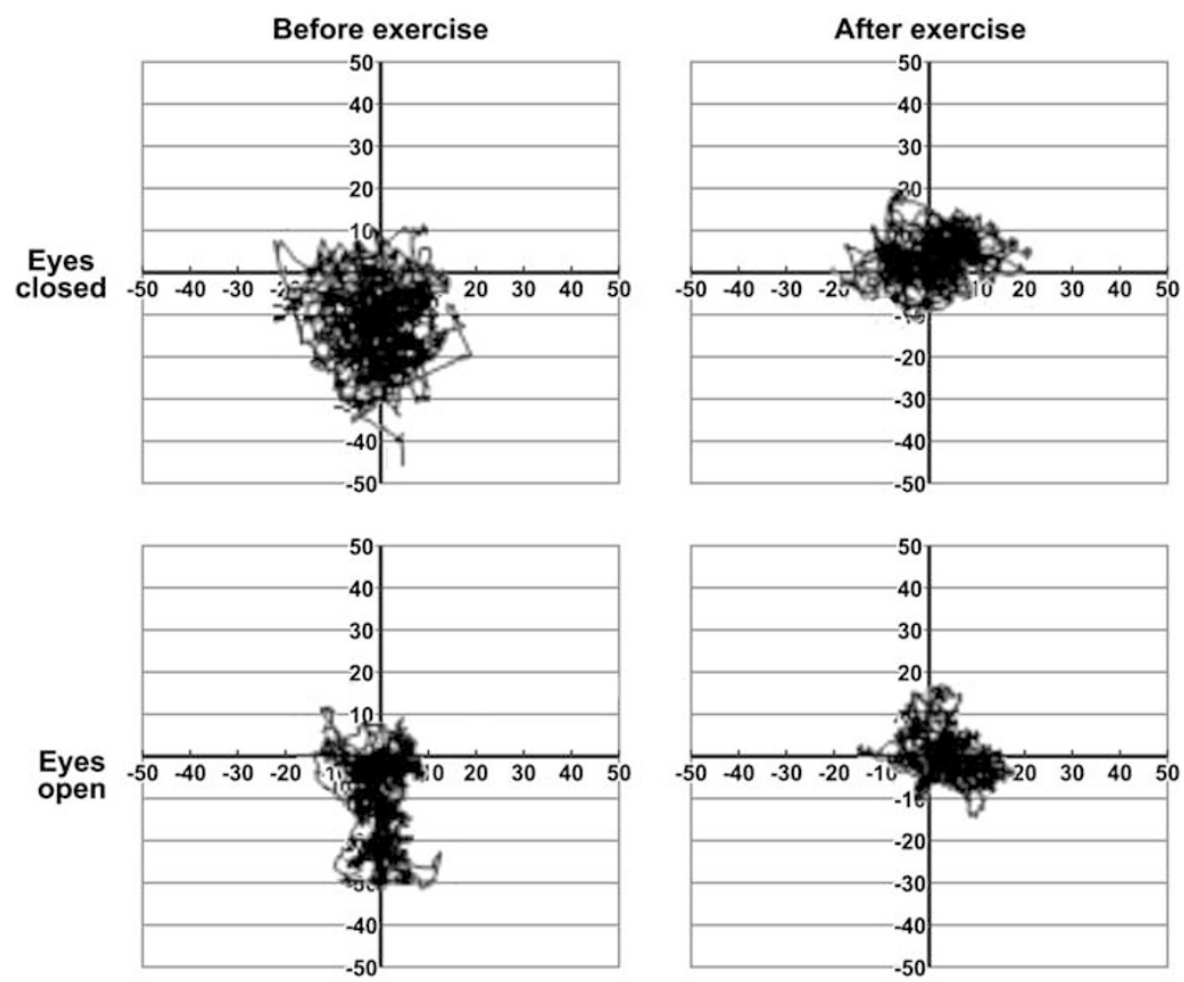

Figure 2 Printouts of data from the trunk control test of body sway with eyes closed (upper) and eyes open (lower) of a 61-year-old woman before exercise (left) and 6 months after exercise (right).

effects of water exercise, and no studies were found regarding the effectiveness of more than 2-3 months of water exercise in improving balance, the impairment of which has been implicated in falls, especially of elderly people.

A previous report showed that water exercise has less of a $\mathrm{BP}$ lowering effect than land-based exercise. ${ }^{15}$ Because of the hybrid nature of the program, it is impossible to determine which element/s were responsible for the positive health results. The study was also limited in that the participants were chosen as a convenience sample, and thus were not randomly assigned to the groups, which resulted in women being overrepresented in the exercise group. Although this may have given some bias to the results, we believe that, taken as a whole, the data support the value of this community-based exercise program. To adequately evaluate the effects of the exercise components, well-designed parallel-control studies would be needed.

The secondary outcome of the study was to determine whether there was a relationship between exercise and improved balance. Such improvement is graphically shown in Figure 2. Notably and importantly, the central gravity of this participant in the exercise group had concentrated and moved forward, both with eyes open and closed, at the 6-month check. Elderly persons often suffer falls that result in fractures of the vertebrae and hips. The study is limited because the design does not allow for the determination of the reason for the change in balance by the exercise group. Further study will need to be conducted to determine whether the improvement was due to improved muscular control in general, the effect of the forward leaning gait necessary when walking in water, or some other component of balance function. To our knowledge, this is the first report of the effectiveness of a hybrid exercise program for improving balance, which is important for reducing falls of the elderly.
The data of the Romberg sign did not show any change between before and after 6 months in the two groups, indicating that the differences of the sway were not related to pathological problems such as the cerebro-cerebellar or peripheral disorders that might cause muscular weakness.

The major findings of this study are improved general health parameters and balance by middle-aged and elderly persons participating in a hybrid exercise program. It is important that most of the participants have continued to exercise independently. This indicates that exercise has become an integral part of their lifestyle, a major goal of the investigators. Programs such as this could easily be made available throughout Japan to older citizens at low cost, with great public health benefits.

\section{Conclusions}

The BP, lipid and glucose metabolism, arterial stiffness, and balance of a group of middle-aged and elderly Japanese were improved by a longterm, comprehensive exercise program incorporating a variety of physical activities.

\section{CONFLICT OF INTEREST}

The authors declare no conflict of interest.

\section{ACKNOWLEDGEMENTS}

This study was supported in part by the Japan Arteriosclerosis Prevention Fund (JAPF) and by Centers of Excellence Program Funding from the Japanese Government. Most of the data used in this paper were previously published in Japanese in Rinsho to Kenkyu (Japanese Journal of Clinical and Experimental Medicne 2007; 84: 402-411), an in-house journal associated with the Kyushu University School of Medical Sciences. We thank Mr Masashi Furuyama for kindly giving his permission to publish the data in English in Hypertension Research. We are indebted to Professor Hiromi Muratani for his support at the medical examinations and valuable comments. 
1 Kiyonaga A, Arakawa K, Tanaka H, Shindo M. Blood pressure and hormoral responses to aerobic exercise. Hypertension 1985; 7: 125-131.

2 Tanaka H, Dinenno FA, Monahan KD, Monaham K, Clevenger CM, DeSouza CA, Seals DR. Aging, habitual exercise, and dynamic arterial compliance. Circulation 2000; 102: 1270-1275.

3 Kodama S, Tanaka S, Saito K, Shu M, Sone Y, Onitake F, Suzuki E, Shimano H, Yamamoto S, Kondo K, Ohashi Y, Yamada N, Sone H. Effect of aerobic execise training on serum levels of high-density lipoprotein cholesterol: a meta-analysis. Arch Intern Med 2007; 167: 999-1008.

4 Arima H, Tanizaki Y, Kiyohara Y, Tsuchihashi T, Kato I, Kubo M, Tanaka K, Ohkubo K, Nakamura H, Abe I, Fujishima M, Iida M. Validity of the JNC VI recommendations for the management of hypertension in a general population of Japanese elderly: the Hisayama study. Arch Intern Med 2003; 163: 361-366.

5 Okayama A, Kadowaki T, Okamura T, Hayakawa T, Ueshima H, The NIPPON80 Research Group. Age-specific effects of systolic and diastolic blood pressures on mortality due to cardiovascular diseases among Japanese men (NIPPON DATA80). J Hypertens 2006; 14: 459-462.

6 Ogihara T, Kikuchi K, Matsuoka H, Fujita T, Higaki J, Horiuchi M, Imai Y, Imaizumi T, Ito $S$, Iwao $H$, Kario $K$, Kawano $Y$, Kim-Mitsuyama S, Kimura G, Katsubara $H$, Matsuura H, Naruse M, Saito I, Shimada K, Shimamoto K, Suzuki H, Takishita S, Tanahashi N, Tsuchihashi T, Uchiyama M, Umemura S, Ishimitsu T, Rakugi H, Japanese Society of Hypertension Committee. The Japanese Society of Hypertension Guidelines for the management of hypertension (JSH 2009). Hypertens Res 2009; 32: 3-107.

7 Yamashina A, Tomiyama H, Takeda K, Tsuda H, Arai T, Hirose K, Koji Y, Hori S, Yamamoto Y. Validity, reproducibility, and clinical significance of noninvasive brachialankle pulse wave velocity measurement. Hypertens Res 2002; 25: 359-364.

8 Tanaka H, Munakata M, Kawano Y, Ohishi M, Shoji T, Sugawara J, Tomiyama H, Yamashina A, Yasuda H, Sawayama T, Ozawa T. Comparison between carotid-femoral and brachial-ankle pulse wave velocity as measures of arterial stiffness. $J$ Hypertens 2009; 27: 2022-2027.

9 Yamashina A, Tomiyama H, Arai T, Hirose K, Koji Y, Hirayama Y, Yamamoto Y, Hori S. Brachial-ankle pulse wave velocity as a marker of atherosclerotic vascular damage and cardiovascular risk. Hypertens Res; 2003; 26: 615-622.

10 Imanishi R, Seto S, Toda G, Yoshida M, Ohtsuru A, Koide Y, Baba T, Yano K. High brachial-ankle pulse wave velocity is an independent predictor of the presence of coronary artery disease in men. Hypertens Res 2004; 27: 71-78.

$11 \mathrm{Im}$ JA, Lee JW, Shim JY, Lee HR, Lee DC. Association between brachial-ankle pulse wave velocity and cardiovascular risk factors in healthy adolescents. J Pediatr 2007; 150: 247-251.

12 Gando Y, Kawano H, Yamamoto K, Sanada K, Tanimoto M, Oh T, Ohmori Y, Miyatani M, Usui C, Takahashi E, Tabata I, Higuchi M, Miyachi M. Age and cardiorespiratory fitness and associated with arterial stiffening and left ventricular remodelling. J Hum Hypertens 2010; 24: 197-206.

13 Tomiyama H, Yamashina A. Non-invasive vascular function tests: their pathophysiological background and clinical application. Circ J 2010; 74: 24-33.

14 Tanaka H. Swimming exercise: impact of aquatic exercise on cardiovascular health. Sports Med 2009; 39: 377-387.
15 Cox KL, Burke V, Beilin LJ, Grove JR, Blanksby BA, Puddey IB. Blood pressure rise with swimming vs. walking in older women: The Sedentary Women Exercise Adherence Trial 2 (SWEAT 2). J Hypertens 2006; 24: 307-314.

16 Lord SR, Ward JA, Williams P, Strudwick M. The effect of a 12-month exercise trial on balance, strength, and falls in older women: a randomized controlled trial. J Am Geriatr Soc 1995; 43: 1198-1206.

17 Grisso JA, Kelsey JL, O'Brien LA, Miles CG, Sidney S, Maislin G, LaPann K, Moritz D, Peters B. Risk factors for hip fracture in men. Hip Fracture Study Group. Am J Epidemiol 1997; 145: 786-793.

18 Myers AH, Young Y, Langlois JA. Prevention of falls in the elderly. Bone 1996; 18 (Suppl): 87S-101S.

19 Hara T, Yoshikawa T, Nakao H, Wang L, Suzuki T, Fujimoto S. The effects of aqua exercise on balance function in middle-aged women. Jpn J Phys Fitness Sports Med 2007; 56: 357-364.

20 Brennan PJ, Greenberg G, Miall WE, Thompson SG. Seasonal variation in arterial blood pressure. Br Med J 1982; 285: 919-923.

21 Kristal-Boneh E, Harari G, Green MS. Circannual variattions in blood cholesterol levels. Chronobiol Int 1993; 10: 37-42.

22 Noda M, Mineyama T, Yamamoto-Honda T. Seasonality in the level of HbAlc. J Chrolonobiol 2010; 16: 59.

23 Kawasaki T, Itoh K, Uezono K, Sasaki H. A simple method for estimating $24 \mathrm{~h}$ urinary sodium and potassium excretion from second morning voiding urine specimen in adults. Clin Exp Pharm Physiol 1993; 20: 7-14.

24 Kawamura M, Kimura K, Takahashi K, Satoh N, Oku K, Adachi T, Nakajima J, Murooka M, Fujiwara T, Hiramori K. Relation of urinary sodium excretion to blood pressure, glucose metabolism, and lipid metabolism in residents of an area of Japan with high sodium intake. Hypertens Res 1997; 20: 287-293.

25 Kawamura M, Kusano Y, Takahashi T, Owada M, Sugawara T. Effectiveness of a spot urine method in evaluating daily salt intake in hypertensive patients taking oral antihypertensive drugs. Hypertens Res 2006; 29: 397-402.

26 Kawasaki T, Kawamura M, Itoh KJ. Second morning urine method developed for estimating daily sodium and potassium excretion. J Metab Clin Nutr 2008; 11: 237-253.

27 Kawamura M, Hashimoto T, Owada M, Sugawara T. The influence of posture on the estimation of daily salt intake by the second morning urine method. Hypertens Res 2010; 33: 505-510.

28 Yamashina A, Tomiyama H, Arai T, Koji Y, Yambe M, Motobe H, Glunizia Z, Yamamoto Y, Hori S. Nomogram of the relation of brachial-ankle pulse wave velocity with blood pressure. Hypertens Res 2003; 26: 801-806.

29 Tomiyama H, Yamashina A, Arai T, Hirose K, Koji Y, Chikamori T, Hori S, Yamamoto Y, Doba N, Hinohara S. Influences of age and gender on results of noninvasive brachialankle pulse wave velocity measurements-a survey of 12517 subjects. Atherosclerosis 2003; 166: 303-309.

30 Cruickshank K, Riste L, Anderson SG, Wright JS, Dunn G, Gosling RG. Aortic pulsewave velocity and its relationship to mortality in diabetes and glucose intolerance: an integrated index of vascular function? Circulation 2002; 106: 2085-2090.

31 Laurent S, Boutouyrie P, Asmar R, Gautier I, Laloux B, Guize L, Ducimetiere P, Benetos A. Aortic stiffness is an independent predictor of all-cause and cardiovascular mortality in hypertensive patients. Hypertension 2001; 37: 1236-1241. 